A Short Proof of Reny's Existence Theorem for Payoff Secure Games

Pavlo Prokopovych (Kyiv School of Economics and Kyiv Economics Institute) 


\title{
A Short Proof of Reny's Existence Theorem for Payoff Secure Games
}

\author{
Pavlo Prokopovych* \\ KSE and KEI, Kyiv, Ukraine
}

December 8, 2008

\footnotetext{
*Email: pprokopo@gmail.com, Phone: 38-044-492-8012, Fax: 38-044-492-8011, Addresses: Kyiv School of Economics, 51 Dehtyarivska, 2nd floor, suite 12, Kyiv 03113, Ukraine; Kyiv Economics Institute, 41 Nyzhniy Val St., Office 55, Kyiv 04070, Ukraine
} 


\begin{abstract}
A short proof of Reny (1999)'s equilibrium existence theorem for payoff secure games is provided. At the heart of the proof lies the concept of a multivalued mapping with the local intersection property. By means of the Fan-Browder collective fixedpoint theorem, we show an approximate equilibrium existence theorem which covers a number of known games. Reny's theorem follows from it straightforwardly.

\section{JEL classification: $\mathrm{C} 72 ; \mathrm{C} 73$}

Keywords: Discontinuous game; Payoff security; Transfer lower semicontinuity; Approximate Nash equilibrium; Reny's theorem
\end{abstract}




\section{Introduction}

Reny (1999)'s theorem states that any game with compact, convex strategy spaces and payoffs at least quasiconcave in each player's strategy has a pure strategy Nash equilibrium if, in addition, the game is better-reply secure. The number of applications of this important result has been steadily growing. So, it has been applied to the problem of mixed strategy equilibrium existence in a nonlinear pricing game by Page and Monteiro (2003) and in a voting game by Carbonell-Nicolau and Ok (2007). Monteiro and Page (2007) provide sufficient conditions for mixed extensions of discontinuous games to be payoff secure.

The focus of this paper is on payoff secure games. We first look in detail at the notion of payoff security. Under the payoff security condition, we associate with each player's payoff function a multivalued mapping that has the local intersection property, which was introduced by Wu and Shen (1996). Then we invoke a generalized version of the Fan-Browder collective fixed-point theorem to show an approximate equilibrium existence theorem (Theorem 2).

Theorem 2 states that every compact, quasiconcave, and payoff secure game has a pure strategy $\varepsilon-$ Nash equilibrium for every $\varepsilon>0$, if, in addition, the players' value functions are continuous. These conditions hold in many economic applications, including first-price sealed-bid auctions with complete information and Bertrand's oligopoly games with different unit costs. It is worth noting that if, in addition to these conditions, limit points of each player's approximate best replies, with the level of approximation converging to zero, are themselves best replies, then the existence of pure strategy Nash equilbria follows (Carmona 2008). 
In order to obtain Reny's theorem for payoff secure games, we approximate from below each player's lower semicontinuous value function (which is the case under payoff security) by an increasing sequence of continuous functions and then invoke Theorem 2.

The next section contains all necessary definitions.

\section{The Model and Some Definitions}

We consider a game $G$ between $n$ players where each player $i$ 's pure strategy set $X_{i}$ is a nonempty compact subset of a Hausdorff topological vector space, and each player $i^{\prime}$ s payoff function $u_{i}$ is a bounded function from $X=\prod_{i \in N} X_{i}$ to $\mathbb{R}$ ( $X$ being endowed with the product topology). Under these conditions, $G=\left(X_{i}, u_{i}\right)_{i=1}^{n}$ is called a compact game. Denote the set of players by $N=\{1, \ldots, n\}$. A game $G=\left(X_{i}, u_{i}\right)_{i=1}^{n}$ is quasiconcave if each $X_{i}$ is convex and $u_{i}\left(\cdot, x_{-i}\right): X_{i} \mapsto \mathbb{R}$ is quasiconcave for all $i \in N$ and all $x_{-i} \in X_{-i}$, where $X_{-i}=\prod_{k \in N \backslash\{i\}} X_{k}$. The graph of $G$ is $\Gamma(G)=\left\{(x, u) \in X \times \mathbb{R}^{n} \mid u_{i}(x)=u_{i}\right.$ for all $\left.i \in N\right\}$. Let $\operatorname{cl} \Gamma(G)$ denote the closure of $\Gamma(G)$ in $X \times \mathbb{R}^{n}$ and $\operatorname{Fr} \Gamma(G)=\operatorname{cl} \Gamma(G) \backslash \Gamma(G)$ the frontier of $\Gamma(G)$, which is the set of points that are in $\operatorname{cl} \Gamma(G)$ but not in $\Gamma(G)$.

Definition 1 Player $i$ can secure a payoff of $\alpha \in \mathbb{R}$ at $x \in X$ if there exists $\bar{x}_{i} \in X_{i}$ such that $u_{i}\left(\bar{x}_{i}, x_{-i}^{\prime}\right) \geq \alpha$ for all $x_{-i}^{\prime}$ in some open neighborhood of $x_{-i}$.

The cornerstone of Reny (1999)'s existence results is the notion of better-reply security.

Definition 2 A game $G=\left(X_{i}, u_{i}\right)_{i=1}^{n}$ is better-reply secure if whenever $\left(x^{*}, u^{*}\right) \in$ $\operatorname{cl} \Gamma(G)$ and $x^{*}$ is not an equilibrium, some player $i$ can secure a payoff strictly above $u_{i}^{*}$ at $x^{*}$. 
Now, we state Reny (1999)'s existence theorem.

Theorem 1 (Reny 1999) If $G=\left(X_{i}, u_{i}\right)_{i=1}^{n}$ is compact, quasiconcave, and betterreply secure, then it possesses a pure strategy Nash equilibrium.

The notion of payoff security, introduced in Reny (1999), is important for applications since many games of economic interest are payoff secure.

Definition 3 A game $G=\left(X_{i}, u_{i}\right)_{i=1}^{n}$ is payoff secure if for every $x \in X$ and every $\varepsilon>0$, each player $i$ can secure a payoff of $u_{i}(x)-\varepsilon$ at $x$.

A notion equivalent to payoff security was introduced independently by Tian (1992).

Definition 4 Let $Z$ and $Y$ be subsets of some topological spaces. A function $f$ : $Z \times Y \mapsto \mathbb{R}$ is $\lambda$-transfer lower semicontinuous in $y$ if for every $(z, y) \in Z \times Y$, $f(z, y)>\lambda$ implies that there exists some point $z^{\prime} \in Z$ and some neighborhood $\mathcal{N}(y)$ of $y$ such that $f\left(z^{\prime}, w\right)>\lambda$ for all $w \in \mathcal{N}(y)$. A function $f: Z \times Y \mapsto \mathbb{R}$ is transfer lower semicontinuous in $y$ if $f$ is $\lambda$-transfer lower semicontinuous in $y$ for every $\lambda \in \mathbb{R}$.

The equivalence of these two notions obtains if, in Definition 4 , we put $f=u_{i}$, $Z=X_{i}, Y=X_{-i}$ and $\lambda=u_{i}(x)-\varepsilon$.

Example 1 in Carmona (2005) shows that a compact, quasiconcave, and payoff secure game need not have a pure strategy Nash equilibrium. A conventional way to ensure the existence of pure strategy Nash equilibria in such games is to add a condition related to but weaker than the upper semicontinuity of the players' payoff functions. Dasgupta and Maskin (1986)'s condition that the sum of the players' payoff functions be upper semicontinuous is often employed. This condition has gone 
through a number of generalizations: reciprocal upper semicontinuity (known also as complimentary discontinuity) by Simon (1987) and then weak reciprocal upper semicontinuity by Bagh and Jofre (2006).

Definition 5 A game $G=\left(X_{i}, u_{i}\right)_{i=1}^{n}$ is weakly reciprocal upper semicontinuous, if for any $(x, \alpha) \in \operatorname{Fr} \Gamma$, there is a player $i$ and $\widehat{x}_{i} \in X_{i}$ such that $u_{i}\left(\widehat{x}_{i}, x_{-i}\right)>\alpha_{i}$.

Payoff security and weak reciprocal upper semicontinuity together imply betterreply security. Under payoff security better-reply security is tantamount to weak reciprocal upper semicontinuity.

We will also need some definitions from multivalued analysis. Let $Y$ and $Z$ denote subsets of some topological spaces. The domain, $\operatorname{Dom} F$, of a multivalued mapping $F: Y \rightarrow Z$ is the subset of elements $y \in Y$ such that $F(y) \neq \varnothing$.

A mapping $F: Y \rightarrow Z$ has open lower sections if $F^{-1}(z)=\{y \in Y: z \in F(y)\}$ is open (maybe empty) in $Y$ for every $z \in Z$.

The concept of a mulivalued mapping with the local intersection property plays a central role for payoff secure games.

Definition 6 A mapping $F: Y \rightarrow Z$ has the local intersection property if for each $y \in \operatorname{Dom} F$, there exists an open neighborhood $\mathcal{N}(y)$ of y such that $\cap_{w \in \mathcal{N}(y)} F(w) \neq \varnothing$.

An important observation is that if $f: Z \times Y \mapsto \mathbb{R}$ is transfer lower semicontinuous in $y$, then the mapping $F_{\lambda}: Y \rightarrow Z$ defined by

$$
F_{\lambda}(y)=\{z \in Z: f(z, y)>\lambda\},
$$

where $\lambda \in \mathbb{R}$, has the local intersection property.

Mappings with the local intersection property have found numerous applications in mathematical economics (see, for example, Mehta and Tarafdar 1987; Baye et al. 
1993). Every nonempty-valued mapping with the local intersection property has a nonempty-valued selection with open lower sections.

Lemma 1 (Ding 1997) If $T: Y \rightarrow Z$ has the local intersection property and $\operatorname{Dom} T=Y$, then there exists a mapping $D: Y \rightarrow Z$ with open lower sections such that $\operatorname{Dom} D=Y$ and $D(y) \subset T(y)$ for all $y \in Y$.

For the reader's convenience, we provide a short proof of Lemma 1 in the Appendix.

\section{$3 \quad$ Payoff Security and Epsilon Equilibria}

In this section we consider conditions which yield pure strategy epsilon equilibria. Theorem 2 was first formulated and proved in an unpublished section of Reny $(1996)^{1}$. However its proof (Reny 1996, p. 16) repeats most of the proof of Reny's main equilibrium existence theorem. Our proof, in its turn, is based on invoking the FanBrowder fixed-point theorem (Theorem 3).

For the sake of completeness, let us remind the definition of a pure strategy $\varepsilon-$ Nash equilibrium. A strategy profile $x \in \Pi_{i \in N} X_{i}$ is a pure strategy $\varepsilon$-Nash equi$\operatorname{librium}(\varepsilon>0)$ of $G=\left(X_{i}, u_{i}\right)_{i=1}^{n}$ if $u_{i}(x) \geq u_{i}\left(y_{i}, x_{-i}\right)-\varepsilon$ for all $y_{i} \in X_{i}$ and all $i \in N$.

It is easy to see that the payoff security of $G$ implies the lower semicontinuity of each player $i$ 's value function $g_{i}: X_{-i} \mapsto \mathbb{R}$ defined by

$$
g_{i}\left(x_{-i}\right)=\sup _{v_{i} \in X_{i}} u_{i}\left(v_{i}, x_{-i}\right)
$$

\footnotetext{
${ }^{1}$ I thank Phil Reny for pointing out this fact to me.
} 
A stronger property often holds, namely, players' value functions are continuous in many games. Examples of such games are first-price sealed-bid auctions with complete information and Bertrand oligopoly games with different unit costs. These games might not possess a pure strategy Nash equilibrium but, by Theorem 2, they always have a pure strategy $\varepsilon-$ Nash equilibrium for every $\varepsilon>0$ because, in addition, they are quasiconcave.

Theorem 2 Let $G=\left(X_{i}, u_{i}\right)_{i=1}^{n}$ is a compact, quasiconcave, and payoff secure game. If, in addition, each player $i$ 's value function $g_{i}$ is continuous, then $G$ possesses a pure strategy $\varepsilon$-Nash equilibrium for every $\varepsilon>0$.

The proof of Theorem 2 is based on invoking the Fan-Browder theorem, which Fan (1966) and Browder (1968) used to show the existence of a pure strategy Nash equilibrium in every compact, quasiconcave, and continuous game.

Theorem 3 (Fan-Browder) Let $X_{1}, \ldots, X_{n}(n \geq 2)$ be nonempty compact convex sets, each in a Hausdorff topological vector space. For each $i \in\{1, \ldots, n\}$, let $M_{i}$ : $X_{-i} \rightarrow X_{i}$ be a mapping with nonempty, convex values and open lower sections. Then there exists $x \in X$ such that $x_{i} \in M_{i}\left(x_{-i}\right)$ for all $i \in N$.

In payoff secure games, players' payoff functions need not be lower semicontinuous in the other players' strategies. So the multivalued mappings associated with players' payoff functions need not have open lower sections. But it turns out that the mappings have the local intersection property, so the following generalization of the Fan-Browder theorem comes in handy.

Corollary 1 Let $X_{1}, \ldots, X_{n}(n \geq 2)$ be nonempty, compact, convex sets, each in a Hausdorff topological vector space. Let $M_{i}: X_{-i} \rightarrow X_{i}$ be a mapping with nonempty, 
convex values and having the local intersection property for each $i \in N$. Then there exists $x \in X$ such that $x_{i} \in M_{i}\left(x_{-i}\right)$ for all $i \in N$.

The proof of Corollary 1 is given in the Appendix. Some other formulations and proofs of Corollary 1 can be found in Wu and Shen (1996, Theorem 2) and Lan and Wu (2002, Theorem 3.1).

Now, we are in the position to prove Theorem 2.

Proof of Theorem 2. Fix $\varepsilon>0$. For each $i \in N$, consider the mapping $M_{i}^{\varepsilon}$ : $X_{-i} \rightarrow X_{i}$ defined by

$$
M_{i}^{\varepsilon}\left(x_{-i}\right)=\left\{x_{i} \in X_{i}: u_{i}\left(x_{i}, x_{-i}\right)>g_{i}\left(x_{-i}\right)-\varepsilon\right\} .
$$

It is clear that $M_{i}^{\varepsilon}$ is nonempty-valued. Since $g_{i}$ is continuous on $X_{-i}$, it is easy to see that $x \mapsto u_{i}\left(x_{i}, x_{-i}\right)-g_{i}\left(x_{-i}\right)$ is transfer lower semicontinuous in $x_{-i}$. Therefore, each $M_{i}^{\varepsilon}$ has the local intersection property. The quasiconcavity of $u_{i}$ in $x_{i}$ implies that $M_{i}^{\varepsilon}\left(x_{-i}\right)$ is convex for every $x_{-i} \in X_{-i}$. Hence, by Corollary 1 , there exists $\bar{x} \in X$ such that $\bar{x}_{i} \in M_{i}^{\varepsilon}\left(\bar{x}_{-i}\right)$ for all $i \in N$.

\section{Proof of Reny's Theorem for Payoff Secure Games}

In this section we prove Theorem 1 for payoff secure games under the assumption that each $X_{i}$ is a nonempty compact subset of a metric vector space. It is useful to note that every metric space is a Hausdorff topological space whose topology is generated by the metric.

Since better reply security is equivalent to weak reciprocal upper semicontinuity under payoff security, Theorem 1 can be reformulated as follows. 
Theorem 4 If $G=\left(X_{i}, u_{i}\right)_{i=1}^{n}$ is a compact, quasiconcave, payoff secure, and weakly reciprocal upper semicontinuous game, then $G$ possesses a pure strategy Nash equilibrium.

On metric spaces, bounded below, lower semicontinuous functions are pointwise limits of increasing sequences of continuous functions (see, for example, Aliprantis and Border 2006, Theorem 3.13). This observation serves as a bridge from Theorem 2 to Theorem 4 . The next lemma shows that any increasing sequence of continuous functions that converges pointwise to a lower semicontinuous function approximates the function "highly enough" for our purposes (see also Reny 1999, Lemma 3.5).

Lemma 2 Let $Z$ be a metric space and let $f: Z \mapsto \mathbb{R}$ be a bounded below, lower semicontinuous, and $\left\{f^{k}\right\}, f^{k}: Z \mapsto \mathbb{R}$, be an increasing sequence of continuous functions such that $\lim _{k} f^{k}(z)=f(z)$ for all $z \in Z$. Then $\liminf _{k} f^{k}\left(z^{k}\right) \geq f(z)$ for all $z \in Z$ and all sequences $\left\{z^{k}\right\}$ in $Z$ converging to $z$.

The proof of Lemma 2 is given in the Appendix.

Now, we proceed to the proof of Theorem 4.

Proof of Theorem 4. The payoff security of $G$ implies that each player $i$ 's value function $g_{i}$ is lower semicontinuous. Moreover, each $g_{i}$ is bounded since the game $G$ is compact. Then there exists an increasing sequence of continuous functions $\left\{g_{i}^{k}\right\}$, $g_{i}^{k}: X_{-i} \mapsto \mathbb{R}$, such that $\lim _{k} g_{i}^{k}\left(x_{-i}\right)=g_{i}\left(x_{-i}\right)$ for all $x_{-i} \in X_{i}$.

Let $\varepsilon^{k}$ be a sequence of positive numbers such that $\varepsilon^{k} \downarrow 0$. For each $i \in N$ and each $k \in\{1,2, \ldots\}$, consider the mapping $M_{i}^{k}: X_{-i} \rightarrow X_{i}$ defined by

$$
M_{i}^{k}\left(x_{-i}\right)=\left\{x_{i} \in X_{i}: u_{i}\left(x_{i}, x_{-i}\right)>g_{i}^{k}\left(x_{-i}\right)-\varepsilon^{k}\right\} .
$$

It is clear that each $M_{i}^{k}$ has nonempty, convex values. Moreover, since $x \mapsto u_{i}\left(x_{i}, x_{-i}\right)-$ 
$g_{i}^{k}\left(x_{-i}\right)$ is transfer lower semicontinuous in $x_{i}$, each $M_{i}^{k}$ has the local intersection property. By Corollary 1 , there exists $x^{k} \in X$ such that $x_{i}^{k} \in M_{i}^{k}\left(x_{-i}^{k}\right)$ for all $i \in N$. Since $X$ is compact, we can assume that the sequence $\left\{x^{k}\right\}$ converges to some $\bar{x} \in X$.

We claim that $\bar{x}$ is a Nash equilibrium of $G$. Assume, by contradiction, that there exists $i^{\prime} \in N$ such that $u_{i^{\prime}}(\bar{x})<g_{i^{\prime}}\left(\bar{x}_{-i^{\prime}}\right)$. Since $\liminf _{k}\left(g_{i^{\prime}}^{k}\left(x_{-i^{\prime}}^{k}\right)-\varepsilon^{k}\right) \geq g_{i^{\prime}}\left(\bar{x}_{-i^{\prime}}\right)$ by Lemma 2, there exists a subsequence $\left\{\bar{x}^{k}\right\}$ of $\left\{x^{k}\right\}$ along which player $i^{\prime \prime}$ s payoff jumps down at $\bar{x}$. The weak reciprocal upper semicontinuity of $G$ implies that some player $i^{\prime \prime}$ can get a payoff strictly above $\alpha_{i^{\prime \prime}}=\liminf _{k}\left(g_{i^{\prime \prime}}^{k}\left(\bar{x}_{-i^{\prime \prime}}^{k}\right)-\varepsilon_{k}\right) \geq g_{i^{\prime \prime}}\left(\bar{x}_{-i^{\prime \prime}}\right)$ at $x=\bar{x}$, a contradiction.

\section{Acknowledgements}

I am grateful to Roy Gardner, Yuriy Gorodnichenko, and Oleksandr Shepotylo for their help.

\section{Appendix}

The appendix contains proofs of some auxiliary results.

\section{Proof of Lemma 1}

First note if $z \in \cap_{y \in \mathfrak{B}} T(y)$ for some neighborhood $\mathfrak{B} \subset Y$, then $\mathfrak{B} \subset T^{-1}(z)$. Define a mapping $D: Y \rightarrow Z$ by $D(y)=\left\{z \in Z: \mathfrak{B} \subset T^{-1}(z)\right.$ for some open neighborhood $\mathfrak{B}$ of $y$ \}. Since $T$ has the local intersection property, $D(y)$ is nonempty for every $y \in Y$. Obviously, $D(y) \subset T(y)$ for every $y \in Y$, and $D^{-1}(z)$ is open (may be empty) for each $z \in Z$. 


\section{Proof of Corollary 1}

Since each $M_{i}$ has the local intersection property and $\operatorname{Dom} M_{i}=X_{-i}$, by Lemma 1 , there exists $F_{i}: X_{-i} \rightarrow X_{i}$ with open lower sections such that Dom $F_{i}=X_{-i}$ and $F_{i}\left(x_{-i}\right) \subset M_{i}\left(x_{-i}\right)$ for every $x_{-i} \in X_{-i}$ and each $i \in N$. Then, by Lemma 5.1 of Yannelis and Prabhakar (1983), the mapping $G_{i}: X_{-i} \rightarrow X_{i}$ defined by $G_{i}\left(x_{-i}\right)=$ $\operatorname{co}_{i}\left(x_{-i}\right)$ also has open lower sections for each $i \in N$ ( $\operatorname{co} A$ denotes the convex hull of $A$ ). By Theorem 3, there exists $x \in X$ such that $x_{i} \in G_{i}\left(x_{-i}\right)$ for all $i \in N$. Since each $M_{i}$ has convex values, $G_{i}\left(x_{-i}\right) \subset M_{i}\left(x_{-i}\right)$ for every $x_{-i} \in X_{-i}$ and each $i \in N$. Therefore, $x_{i} \in M_{i}\left(x_{-i}\right)$ for all $i \in N$.

\section{Proof of Lemma 2}

Suppose, by way of contradiction, that there exist $z^{0} \in Z$ and a sequence $\left\{z^{k}\right\}$ in $Z$ converging to $z^{0}$ such that $\lim _{k} f^{k}\left(z^{k}\right)<f\left(z^{0}\right)$. Since $\lim _{k} f^{k}\left(z^{0}\right)=f\left(z^{0}\right)$ and $\left\{f^{k}\right\}$ is an increasing sequence, there exist integer $m$ and a small enough $\varepsilon>0$ such that

$f^{k}\left(z^{0}\right)>\lim _{k} f^{k}\left(z^{k}\right)+\varepsilon$ for all $k \geq m$. The continuity of $f^{m}$ implies that there exists a neighborhood $U$ of $z^{0}$ such that $f^{m}(z)>\lim _{k} f^{k}\left(z^{k}\right)+\varepsilon$ for all $z \in U$. Since $\left\{f^{k}\right\}$ is an increasing sequence, $f^{k}(z)>\lim _{k} f^{k}\left(z^{k}\right)+\varepsilon$ for all $z \in U$ and all $k \geq m$, a contradiction.

\section{References}

Aliprantis CD, Border KC (2006) Infinite Dimensional Analysis, A Hitchhiker's Guide, 3rd ed. Springer-Verlag, Berlin.

Bagh A, Jofre A (2006) Reciprocal Upper Semicontinuity and Better Reply Secure Games: a Comment, Econometrica 74:1715-1751 
Baye MR, Tian G, Zhou, J (1993) Characterization of the Existence of Equilibria in Games with Discontinuous and Non-quasiconcave Payoffs. Review of Economics Studies 60:935-948

Browder F (1968) The Fixed Point Theory of Multi-valued Mappings in Topological Vector Spaces, Mathematische Annalen 177:283-301

Carbonell-Nicolau O, Ok EA (2007) Voting over Income Taxation, Journal of Economic Theory 134:249-286

Carmona G (2005) On the Existence of Equilibria in Discontinuous Games: Three Counterexamples, International Journal of Game Theory 33:181-187

Carmona G (2008) An Existence Result for Discontinuous Games, Mimeo, Universidade Nova de Lisboa.

Dasgupta P, Maskin E (1986) The Existence of Equilibrium in Discontinuous Economic Games, I: Theory, Review of Economics Studies 53:1-26

Ding XP (1997) A Coincidence Theorem Involving Contractible Spaces, Applied Mathematics Letters 10:53-56

Fan K (1966) Applications of a Theorem Concerning Sets with Convex Sections, Mathematische Annalen 163:189-203

Lan KQ, Wu JH (2002) A Fixed-Point Theorem and Applications to Problems on Sets with Convex Sections and to Nash Equilibria, Mathematical and Computer Modelling 36:139-145

Mehta G, Tarafdar E (1987) Infinite-Dimensional Gale-Nikaido-Debreu Theorem and a Fixed-Point Theorem of Tarafdar, Journal of Economic Theory 41:333-339

Page FH, Monteiro PK (2003) Three Principles of Competitive Nonlinear Pricing. Journal of Mathematical Economics 39:63-109

Monteiro PK, Page, FH (2007) Uniform Payoff Security and Nash Equilibrium in Compact Games, Journal of Economic Theory 134:566-575 
Reny PJ (1996) Local Payoff Security and the Existence of Pure and Mixed Strategy Nash Equilibria in Discontinuous Games, Mimeo, University of Pittsburgh.

Reny PJ (1999) On the Existence of Pure and Mixed Strategy Nash Equilibria in Discontinuous Games, Econometrica 67:1029-1056

Simon L (1987) Games with Discontinuous Payoffs, Review of Economics Studies 54:569-597

Tian G (1992) Generalizations of the FKKM Theorem and the Ky Fan Minimax Inequality, with Applications to Maximal Elements, Price Equilibrium, and Complementarity, Journal of Mathematical Analysis and Applications 170:457-471

Wu X, Shen S (1996) A Further Generalization of Yannelis-Prabhakar's Continuous Theorem and its Applications. Journal of Mathematical Analysis and Applications 197:61-74

Yannelis NC, Prabhakar ND (1983) Existence of Maximal Elements and Equilibria in Linear Topological Spaces, Journal of Mathematical Economics 12:233-245 